\title{
Trece registros nuevos de Lutzomyia (Diptera: Psychodidae) para el departamento de Vichada, Orinoquia Colombiana
}

\author{
Rafael José Vivero ${ }^{1,5}$, Eduar Elías Bejarano ${ }^{2}$, Mirley Castro ${ }^{3}$, Andrés Vélez, , Juan Esteban Pérez, \\ Alveiro Pérez-Doria ${ }^{2}$, Iván Darío Vélez ${ }^{1} \&$ Lina María Carrillo ${ }^{1,4}$ \\ ${ }^{1}$ Programa de Estudio y Control de Enfermedades Tropicales - PECET, Universidad de Antioquia, \\ Calle 62 No. 52-69, A.A. 1226, Medellín, Colombia \\ ${ }^{2}$ Grupo de Investigaciones Biomédicas, Universidad de Sucre, \\ Cra. 14 No. 16 B-32, A.A. 406, Sincelejo, Colombia \\ ${ }^{3}$ Secretaría de Salud de Vichada, \\ Av. Orinoco, Calle 10, Puerto Carreño, Colombia \\ ${ }^{4}$ Facultad de Ciencias Agrarias, Escuela de Medicina Veterinaria, Universidad de Antioquia, \\ Calle 62 No. 52-69, A.A. 1226, Medellín, Colombia \\ ${ }^{5}$ Autor para correspondencia: Rafael José Vivero, e-mail: rajovigo2001@yahoo.com
}

VIVERO, R.J., BEJARANO, E.E., CASTRO, M., VÉLEZ, A., PÉREZ, J.E., PÉREZ-DORIA, A., VÉLEZ, I.D. \& CARRILLO, L.M. Thirteen new records of Lutzomyia (Diptera: Psychodidae) for the department of Vichada, Colombian Orinoquia. Biota Neotrop. 10(2): http://www.biotaneotropica.org.br/v10n2/en/abstract?shortcommunication+bn01510022010.

\begin{abstract}
Lutzomyia trinidadensis (Newstead, 1922), L. bettinii Feliciangeli, Ramírez-Pérez \& Ramírez, 1988, L. flaviscutellata (Mangabeira, 1942), L. yuilli Young \& Porter, 1972, L. saulensis (Floch \& Abonnenc, 1944), L. runoides (Fairchild \& Hertig, 1953), L. ayrozai (Barretto \& Coutinho, 1940), L. claustrei Abonnenc, Leger \& Fauran, 1979, L. walkeri (Newstead, 1914), L. preclara Young \& Arias, 1984, L. (geniculata) sp., L. (squamiventris) sp., and $L$. (Evandromyia) sp., are recorded for the first time in the department of Vichada, Colombia. In addition, the presence of L. antunesi (Coutinho, 1939) and L. aragaoi (Costa Lima, 1932) in this department is confirmed. Sand flies were collected in the municipalities of Puerto Carreño, Cumaribo, and La Primavera, using CDC light traps, Shannon trap, and human bait, and by active search in resting places. The epidemiological importance of these sand fly species is discussed in relation with the cutaneous leishmaniasis.
\end{abstract}

Keywords: Sand flies, leishmaniasis, Lutzomyia, Orinoquia, Colombia.

VIVERO, R.J., BEJARANO, E.E., CASTRO, M., VÉLEZ, A., PÉREZ, J.E., PÉREZ-DORIA, A., VÉLEZ, I.D. \& CARRILLO, L.M. Trece registros nuevos de Lutzomyia (Diptera: Psychodidae) para el departamento de Vichada, Orinoquia Colombiana. Biota Neotrop. 10(2): http://www.biotaneotropica.org.br/v10n2/es/ abstract?short-communication+bn01510022010.

Resumen: Se informa el primer hallazgo de Lutzomyia trinidadensis (Newstead, 1922), L. bettinii Feliciangeli, Ramírez-Pérez \& Ramírez, 1988, L. flaviscutellata (Mangabeira, 1942), L. yuilli Young \& Porter, 1972, L. saulensis (Floch \& Abonnenc, 1944), L. runoides (Fairchild \& Hertig, 1953), L. ayrozai (Barretto \& Coutinho, 1940), L. claustrei Abonnenc, Leger \& Fauran, 1979, L. walkeri (Newstead, 1914), L. preclara Young \& Arias, 1984, L. (geniculata) sp., L. (squamiventris) sp., y L. (Evandromyia) sp., en el departamento de Vichada, Colombia. También se confirma la presencia de L. antunesi (Coutinho, 1939) y L. aragaoi (Costa Lima, 1932) en este departamento. Los flebotomíneos fueron recolectados en los municipios de Puerto Carreño, Cumaribo, y La Primavera, con trampas de luz tipo CDC, trampa Shannon y atrayente humano, y mediante búsqueda activa en lugares de reposo. Se discute la importancia epidemiológica de estas especies de flebotomíneos en relación con la leishmaniosis cutánea.

Palabras-clave: Flebotomíneos, leishmaniosis, Lutzomyia, Orinoquia, Colombia. 


\section{Introducción}

Colombia es uno de los países denominados megadiversos, por albergar una enorme cantidad y diversidad de animales y plantas. La diversidad de la fauna colombiana, incluye a los insectos transmisores de microorganismos patógenos, dentro de los cuales se encuentran los flebotomíneos, vectores naturales de parásitos del género Leishmania Ross, 1903. La infección por estos parásitos produce, en humanos, diversos cuadros clínicos, cutáneos, mucocutáneos o viscerales, conocidos como leishmaniosis (Lainson \& Shaw 2005).

En América, Colombia ocupa el segundo lugar en diversidad de flebotomíneos, con más de 160 especies reconocidas en diferentes unidades biogeográficas del país (Bejarano 2006), pero los departamentos de la Amazonia y Orinoquia no han sido exhaustivamente muestreados. Dentro de la Orinoquia, se encuentra el departamento de Vichada, donde sólo están registradas tres especies de Lutzomyia, L. antunesi (Coutinho, 1939), L. aragaoi (Costa Lima, 1932) y L. sp. de Baduel (Floch \& Abonnenc, 1945) (Bejarano 2006), aunque conforma el segundo departamento más extenso de Colombia. Esto contrasta con el número de especies de flebotomíneos encontrado en departamentos colindantes, que presentan una composición ecológica similar, como Guainía con nueve especies (Bejarano et al. 2007, Flores \& Ferro 2007), Meta con 35 especies (Bejarano 2006, Vásquez-Trujillo et al. 2008) y Guaviare con 37 especies (Cabrera et al. 2009).

La abundancia y amplia diversidad de especies en localidades vecinas del departamento de Vichada, evidencia el desconocimiento de la fauna flebotomínea para esta área de la Orinoquia Colombiana, posiblemente, por la falta de exploración asociada a la dificultad de acceso a la zona. El presente trabajo tiene como objetivo registrar el hallazgo de trece especies del género Lutzomyia en el departamento de Vichada.

\section{Materiales y Métodos}

La recolección de los flebotomíneos se realizó en el departamento

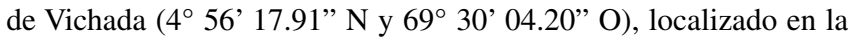
margen izquierda del Río Orinoco y derecha del Río Meta, en la llamada altillanura. En la cabecera municipal de Puerto Carreño se registra en promedio por año una temperatura de $28^{\circ} \mathrm{C}$ y un rango de precipitación anual que va de los 2.000 a $4.000 \mathrm{~mm}$, que corresponde, según la clasificación de Holdridge (1967), a bosque húmedo tropical (bh-T), con una altitud comprendida entre 51 y $161 \mathrm{msnm}$ (IGAC 1996). En algunas de las localidades muestreadas (Caño Ariba) se denota una cobertura vegetal intervenida por cultivos ilícitos cercanos al peridomicilio, con cambios graduales al aproximarse al fragmento de bosque mas conservado.

En la zona de estudio se efectuaron muestreos esporádicos por el Programa de Vigilancia Entomológica de la Secretaría de Salud de Vichada y el Programa de Estudio y Control de Enfermedades Tropicales (PECET) de la Universidad de Antioquia, durante los años 2007 y 2008, en los municipios de Puerto Carreño (La Primavera y Gaitán), Cumaribo (Caño Ariba, Santa Rita-El Sejal, Parque Nacional Natural El Tuparro) y La Primavera, donde la leishmaniosis cutánea es endémica.

En la mayoría de las localidades se trabajó entre una y dos noches utilizando trampas de luz tipo CDC, ubicadas a 1,3 m de altura en ambientes peri-extradomiciliares. Adicionalmente, en la localidad de Caño Ariba se usó una trampa Shannon en el peridomicilio, la cual fue operada de las 18:00 a las 21:00 por dos personas, y en el casco urbano de Puerto Carreño se desarrolló, entre las 06:00 y 07:00, una búsqueda de flebotomíneos en sitios potenciales de reposo cercanos a viviendas. También en los municipios de Puerto Carreño y La
Primavera se recolectaron los flebotomíneos que, ocasionalmente, intentaron picar a humanos.

Los ejemplares capturados se almacenaron en tubos de $1,5 \mathrm{~mL}$ a una temperatura de $-20{ }^{\circ} \mathrm{C}$. Cada flebotomíneo fue aclarado en lactofenol y montado en láminas portaobjeto con el medio de Hoyer. La determinación taxonómica se llevó a cabo con las claves de Young \& Duncan (1994) y Galati (2009). Estos montajes están distribuidos en la colecciones de flebotomíneos de la Secretaría de Salud de Vichada, el PECET de la Universidad de Antioquia y el Grupo de Investigaciones Biomédicas de la Universidad de Sucre.

\section{Resultados y Discusión}

Se recolectaron en total 182 flebotomíneos pertenecientes a 15 especies de Lutzomyia, 13 de las cuales constituyen primeros registros para el departamento de Vichada, L. trinidadensis (Newstead, 1922), L. bettinii Feliciangeli, Ramírez-Pérez \& Ramírez, 1988, L. flaviscutellata (Mangabeira, 1942), L. yuilli Young \& Porter, 1972, L. saulensis (Floch \& Abonnenc, 1944), L. runoides (Fairchild \& Hertig, 1953), L. ayrozai (Barretto \& Coutinho, 1940), L. claustrei Abonnenc, Leger \& Fauran, 1979, L. walkeri (Newstead, 1914), L. preclara Young \& Arias, 1984, L. (geniculata) sp., L. (squamiventris) sp., y L. (Evandromyia) sp., y dos habían sido documentadas antes en la zona, L. antunesi y L. aragaoi. El número absoluto de individuos recolectados de cada especie discriminado por sexo, municipio y método de muestreo, se presenta en la Tabla 1.

Dentro de las especies de importancia médica, sobresalen L. antunesi, L. yuilli y L. flaviscutellata, del subgénero Nyssomyia Barretto 1962, relacionadas previamente con el ciclo epidemiológico de la leishmaniosis cutánea, y se destaca L. antunesi por la densidad relativamente alta, cercana al $42 \%$, y presencia en la mayoría de las localidades muestreadas. Un estudio reciente de determinación de infección natural en el departamento de Meta, Colombia, asoció a L. antunesi con la trasmisión de Leishmania spp. (Vásquez-Trujillo et al. 2008). L. yuilli está incriminada como vector de Le. panamensis Lainson \& Shaw, 1972, en el departamento de Boyacá, piedemonte del valle del Magdalena medio, Colombia (Santamaría et al. 2006), mientras que L. flaviscutellata es un reconocido transmisor de Le. amazonensis Lainson \& Shaw, 1972, en Brasil (Rangel \& Lainson 2009) y probablemente también en Colombia.

La segunda especie más abundante en el departamento de Vichada fue $L$. trinidadensis, que ha sido encontrada infectada en la naturaleza con Le. venezuelensis Bonfante-Garrido, 1980, en Venezuela (Bonfante et al. 1990). Desde el punto de vista epidemiológico, es importante resaltar también el hallazgo de cuatro miembros del subgénero Psychodopygus Mangabeira, 1941, taxón reconocido por albergar especies con un marcado comportamiento antropofílico. L. ayrozai y L. claustrei están incriminadas como vectores de Le. naiffi Lainson \& Shaw, 1989, en Brasil (Rangel \& Lainson 2003), en tanto que las especies de la serie $L$. squamiventris revisten importancia como transmisores de Le. braziliensis Vianna, 1911 (Rangel \& Lainson 2003), aunque por lo general las hembras de las especies de esta serie no se pueden distinguir entre sí. De forma similar, la otra hembra hallada del subgénero Psychodopygus pertenece al complejo L. geniculata, taxón informal cuyos integrantes están aún por discriminar.

Durante el estudio también se recolectaron especies no relacionadas hasta ahora con el ciclo epidemiológico de la leishmaniosis, como L. bettinii, L. saulensis, L. runoides, L. aragaoi, L. walkeri y L. preclara. Esta última estaba registrada sólo en los departamentos de Meta y Amazonas (Bejarano 2006), mientras que L. bettinii había sido informada únicamente para el departamento de Guainía (Flores \& Ferro 2007). 
Tabla 1. Especie, sexo, método de muestreo y número absoluto de los flebotomíneos del género Lutzomyia recolectados en los municipios de Puerto Carreño, Cumaribo y La Primavera, Departamento de Vichada, Colombia.

Table 1. Species, sex, sampling method, and absolute number of sand flies of the genus Lutzomyia collected in the municipalities of Puerto Carreño, Cumaribo, and La Primavera, Department of Vichada, Colombia.

\begin{tabular}{|c|c|c|c|c|c|c|c|c|c|c|c|}
\hline \multirow[t]{3}{*}{ Especie } & \multicolumn{4}{|c|}{ Cumaribo } & \multicolumn{5}{|c|}{ Puerto Carreño } & \multirow{3}{*}{$\begin{array}{c}\text { La Primavera } \\
\text { Cebo Humano } \\
q\end{array}$} & \multirow[t]{3}{*}{ Total } \\
\hline & \multicolumn{2}{|c|}{ CDC } & \multicolumn{2}{|c|}{ Shannon } & \multicolumn{2}{|c|}{$\mathrm{CDC}$} & \multirow{2}{*}{$\begin{array}{c}\text { Cebo Humano } \\
q\end{array}$} & \multicolumn{2}{|c|}{ Reposo } & & \\
\hline & 우 & $\hat{0}$ & 우 & $\hat{0}$ & q & 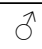 & & ㅇ & 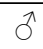 & & \\
\hline L. antunesi & 38 & 29 & 2 & 5 & 1 & 1 & - & 1 & 1 & - & 78 \\
\hline L. trinidadensis & 18 & 14 & - & - & 1 & - & 1 & 1 & - & - & 35 \\
\hline L. bettinii & 3 & 6 & 4 & - & - & - & - & - & - & - & 13 \\
\hline L. flaviscutellata & 12 & - & - & - & - & - & - & - & - & - & 12 \\
\hline L. yuilli & 6 & - & - & - & - & - & - & - & - & - & 6 \\
\hline L. saulensis & 6 & - & - & - & - & - & - & - & - & - & 6 \\
\hline L. runoides & 5 & 1 & - & - & - & - & - & - & - & - & 6 \\
\hline L. aragaoi & 2 & - & 1 & - & - & - & - & - & - & - & 3 \\
\hline L. ayrozai & 2 & - & - & - & - & - & - & - & - & - & 2 \\
\hline L. claustrei & 1 & - & - & - & - & - & - & - & - & - & 1 \\
\hline L. walkeri & - & 1 & - & - & - & - & - & - & - & - & 1 \\
\hline L. preclara & - & 1 & - & - & - & - & - & - & - & - & 1 \\
\hline L. (geniculata) sp. & - & 1 & - & - & - & - & - & - & - & - & 1 \\
\hline L. (squamiventris) sp. & - & - & - & - & - & - & - & - & - & 17 & 17 \\
\hline L. (Evandromyia) sp. & 6 & - & - & - & - & - & - & - & - & - & 6 \\
\hline Total & 99 & 53 & 7 & 5 & 2 & 1 & 1 & 2 & 1 & 17 & 188 \\
\hline
\end{tabular}

Las condiciones del ecosistema donde se realizaron los muestreos, permite suponer que la relación de los flebotomíneos con la aparición de casos de leishmaniosis en Vichada podría estar definida por varios factores. La deforestación, que ejerce un efecto negativo en la abundancia y diversidad de los flebotomíneos y expone a la población humana a la picadura de las especies antropofílicas aumentando la posibilidad de infectarse (Travi et al. 2002, Silva et al. 2007). Así mismo, actividades diarias necesarias para el sostenimiento de la población, como la agricultura y la migración fluvial, también podrían influir directamente en la aparición esporádica o en el establecimiento de un foco de leishmaniosis cutánea en esta área de la Orinoquia.

En conclusión, se reconoce para el departamento de Vichada, la existencia de 15 especies del género Lutzomyia, trece de ellas como primer registro y se confirma la presencia de L. antunesi y L. aragaoi. Esto expone la necesidad de desarrollar estudios eco-epidemiológicos con el fin de fijar el papel de las especies halladas en la diseminación de agentes patógenos que afectan al humano en la región.

\section{Agradecimientos}

A Juan Eduardo Gómez, Juan Carlos Durán y Adriana Betancourt del Programa de Enfermedades Transmitidas por Vectores, de Vichada, por su colaboración durante el desarrollo del estudio. Esta investigación se realizó dentro del marco del convenio celebrado entre la Secretaría de Salud de Vichada y el Programa de Estudio y Control de Enfermedades Tropicales (PECET) de la Universidad de Antioquia.

\section{Referencias Bibliográficas}

BEJARANO, E.E. 2006. Lista actualizada de los psicódidos (Diptera: Psychodidae) de Colombia. Folia Entomol. 45(1):47-56.

BEJARANO, E.E., CASTRO, M., PÉREZ-DORIA, A., HERNÁNDEZOVIEDO, E., VÉLEZ, A. \& VÉLEZ, I.D. 2007. Primer Informe de
Lutzomyia França en el Departamento de Guainía, Amazonia Colombiana, y de Brumptomyia mesai Sherlock (Diptera: Psychodidae) en el Litoral Caribe Colombiano. Neotrop. Entomol. 36(6):990-993.

BONFANTE, G., URDANETA, R. \& ALVARADO, I. 1990. Natural infection of Lutzomyia trinidadensis (Diptera, Psychodidae) with Leishmania in Barquisimeto, Venezuela. Mem. Inst. Oswaldo Cruz 85(4):477.

CABRERA, O.L., MOSQUERA, L., SANTAMARÍA, E. \& FERRO, C. 2009. Flebótomos (Diptera: Psychodidae) del departamento de Guaviare, Colombia, con nuevos registros para el país. Biomédica 29(1):73-86.

FLORES, L. \& FERRO, C. 2007. Especies de Lutzomyia (Diptera:Psycodidae) encontradas en el municipio de Inírida, departamento de Guainía, Colombia. Biomédica 27(2):222.

GALATI, E.A.B. 2009. Phlebotominae (Diptera, Psychodidae). Classificação morfologia e terminologia e identificação de adultos. Universidade de São Paulo, São Paulo. Apostila disciplina HEP 5752: Bioecologia e identificação de Phlebotominae.

HOLDRIDGE, L.R. 1967. Life zone ecology. Tropical Science Center, San José.

INSTITUTO GEOGRÁFICO AGUSTÍN CODAZZI - IGAC. 1996. Diccionario geográfico de Colombia. 3 ed. Instituto Geográfico Agustín Codazzi, Santa Fé de Bogotá.

LAINSON, R. \& SHAW, J.J. 2005. New world leishmaniasis. In Parasitology (F.E.G. Cox, D. Wakelin, S.H. Gillespie \& D. Despommier, eds.). 10 ed. Hodder Arnold, London, p.313-349.

RANGEL, E.F. \& LAINSON, R. 2003. Ecologia das leishmanioses: transmissores de leishmaniose tegumentar americana. In Flebotomíneos do Brasil (E.F. Rangel \& R. Lainson, eds.). Editora Fiocruz, Rio do Janeiro, p.291-310.

RANGEL, E.F. \& LAINSON, R. 2009. Proven and putative vectors of American cutaneous leishmaniasis in Brazil: aspects of their biology and vectorial competence. Mem. Inst. Oswaldo Cruz 104(7):937-954. 
SANTAMARÍA, E., PONCE, N., ZIPA, Y. \& FERRO, C. 2006 Presencia en el peridomicilio de vectores infectados con Leishmania (Viannia) panamensis en dos focos endémicos en el occidente de Boyacá, piedemonte del valle del Magdalena medio, Colombia. Biomédica 26(1):82-94.

SILVA, D.F., FREITAS, R.A. \& FRANCO, M.R. 2007. Diversidade e abundância de flebotomíneos do gênero Lutzomyia (Diptera: Psychodidae) em áreas de mata do nordeste de Manacapuru, AM. Neotrop. Entomol. 36(1):138-144.

TRAVI, B.L, ADLER, G.H., LOZANO, M., CADENA, H. \& MONTOYALERMA, J. 2002. Impact of habitat degradation on Phlebotominae (Diptera: Psychodidae) of tropical dry forest in Northern Colombia. J. Med. Entomol. 39(3):451-456.
VÁSQUEZ-TRUJILLO, A., SANTAMARÍA-HERREÑO, E., GONZÁLEZREINA, A.E., BUITRAGO-ÁLVAREZ, L.S., GÓNGORA-ORJUELA, A. \& CABRERA-QUINTERO, O.L. 2008. Lutzomyia antunesi, probable vector de leishmaniasis cutánea en el área rural de Villavicencio. Rev. Salud Pública. 10(4):625-632.

YOUNG, D.G. \& DUNCAN, M.A. 1994. Guide to the identification and geographic distribution of Lutzomyia sand flies in Mexico, the West Indies, Central and South America (Diptera: Psychodidae). Mem. Am. Entomol. Inst. 54:1-881.

Recibido en 11/01/10 Versión revisada recibida en $26 / 03 / 10$ Aceptado en 30/04/10 